\title{
Perioperative Rosuvastatin in Cardiac Surgery
}

\author{
Zhe Zheng, M.D., Raja Jayaram, M.D., Lixin Jiang, M.D., \\ Jonathan Emberson, Ph.D., Yan Zhao, M.D., Qi Li, M.D., Juan Du, M.D., \\ Silvia Guarguagli, M.D., Michael Hill, D.Phil., Zhengming Chen, D.Phil., \\ Rory Collins, M.B., B.S., and Barbara Casadei, M.D., D.Phil.
}

From the Department of Cardiovascular Surgery, State Key Laboratory of Cardiovascular Disease, National Clinical Research Center of Cardiovascular Diseases, Fuwai Hospital, National Center for Cardiovascular Diseases, Chinese Academy of Medical Sciences and Peking Union Medical College, Beijing (Z.Z., L.J., Y.Z., Q.L., J.D., Z.C.); and the Division of Cardiovascular Medicine, Radcliffe Department of Medicine (R.J., S.G., B.C.), and the Clinical Trial Service Unit and Epidemiological Studies Unit, Nuffield Department of Population Health (J.E., M.H., Z.C., R.C.), University of Oxford, Oxford, United Kingdom. Address reprint requests to Dr. Zheng at the Department of Cardiovascular Surgery, Fuwai Hospital, National Center for Cardiovascular Diseases, Chinese Academy of Medical Sciences and Peking Union Medical College, 167 Beilishi Rd., Xicheng District, Beijing 100037, China, or at zhengzhe@fuwai.com or to Dr. Casadei at the Division of Cardiovascular Medicine, Radcliffe Department of Medicine, University of Oxford, L6, West Wing, John Radcliffe Hospital, Oxford OX3 9DU, United Kingdom, or at barbara.casadei@cardiov.ox.ac.uk.

N EngIJ Med 2016;374:1744-53. DOI: 10.1056/NEJMoal507750

Copyright @ 2016 Massachusetts Medical Society.
ABSTRACT

\section{BACKGROUND}

Complications after cardiac surgery are common and lead to substantial increases in morbidity and mortality. Meta-analyses of small randomized trials have suggested that perioperative statin therapy can prevent some of these complications.

\section{METHODS}

We randomly assigned 1922 patients in sinus rhythm who were scheduled for elective cardiac surgery to receive perioperative rosuvastatin (at a dose of $20 \mathrm{mg}$ daily) or placebo. The primary outcomes were postoperative atrial fibrillation within 5 days after surgery, as assessed by Holter electrocardiographic monitoring, and myocardial injury within 120 hours after surgery, as assessed by serial measurements of the cardiac troponin I concentration. Secondary outcomes included major in-hospital adverse events, duration of stay in the hospital and intensive care unit, left ventricular and renal function, and blood biomarkers.

\section{RESULTS}

The concentrations of low-density lipoprotein cholesterol and C-reactive protein after surgery were lower in patients assigned to rosuvastatin than in those assigned to placebo $(\mathrm{P}<0.001)$. However, the rate of postoperative atrial fibrillation did not differ significantly between the rosuvastatin group and the placebo group (21.1\% and 20.5\%, respectively; odds ratio 1.04 ; $95 \%$ confidence interval [CI], 0.84 to $1.30 ; \mathrm{P}=0.72$ ), nor did the area under the troponin I-release curve (102 $\mathrm{ng} \times$ hour per milliliter and $100 \mathrm{ng} \times$ hour per milliliter, respectively; between-group difference, $1 \% ; 95 \% \mathrm{CI},-9$ to $13 ; \mathrm{P}=0.80$ ). Subgroup analyses did not indicate benefit in any category of patient. Rosuvastatin therapy did not result in beneficial effects on any of the secondary outcomes but was associated with a significant absolute $( \pm$ SE) excess of $5.4 \pm 1.9$ percentage points in the rate of postoperative acute kidney injury $(\mathrm{P}=0.005)$.

\section{CONCLUSIONS}

In this trial, perioperative statin therapy did not prevent postoperative atrial fibrillation or perioperative myocardial damage in patients undergoing elective cardiac surgery. Acute kidney injury was more common with rosuvastatin. (Funded by the British Heart Foundation and others; STICS ClinicalTrials.gov number, NCT01573143.) 
D

ESPITE ADVANCES IN SURGICAL AND perioperative care, postoperative complications after cardiac surgery remain frequent, leading to substantial increases in mortality, morbidity, and costs. ${ }^{1}$ Inflammation and oxidative stress have been implicated in the pathogenesis of atrial fibrillation and other postoperative complications of cardiac surgery. ${ }^{2}$ In particular, the incidence of postoperative atrial fibrillation coincides with the peak of the systemic inflammatory response after cardiac surgery, ${ }^{2}$ is independently associated with atrial markers of oxidative stress, ${ }^{3,4}$ and has been reported to be partially prevented by antiinflammatory drugs. .,6 $^{5}$

Statins have been shown to have rapid antiinflammatory and antioxidant effects ${ }^{7}$; for example, 3 days of preoperative treatment with atorvastatin was associated with a lower production of reactive oxygen species, as compared with placebo, in atrial and vascular samples obtained from patients undergoing cardiac surgery, ${ }^{4,8}$ and the initiation of statin therapy shortly before cardiac surgery has been shown to decrease the concentration of C-reactive protein in some studies ${ }^{9-12}$ but not all. ${ }^{13-16}$ In keeping with these findings, perioperative statin therapy (typically starting in the week before surgery) has been associated with a lower incidence of atrial fibrillation after cardiac surgery, as well as less severe myocardial injury, preserved left ventricular and renal function, and shorter stays in the intensive care unit (ICU) and hospital. .,12,17,18

On the basis of this evidence, practice guidelines currently recommend perioperative statin therapy for the prevention of atrial fibrillation and other in-hospital complications after cardiac surgery. ${ }^{19-21}$ However, these recommendations are based on randomized trials that were small and had other important limitations (Table S1 in the Supplementary Appendix, available with the full text of this article at NEJM.org). The Statin Therapy in Cardiac Surgery (STICS) trial was a randomized, placebo-controlled trial that aimed to provide more definitive evidence regarding the effects of perioperative statin therapy on postoperative complications.

\section{METHODS}

\section{TRIAL OVERSIGHT}

The trial protocol, available at NEJM.org, was designed by all the authors and was approved by the ethics committees at Fuwai Hospital in Beijing (where patients were recruited) and at the University of Oxford in the United Kingdom (where all electrocardiographic, blood, and statistical analyses were performed). All the authors contributed to the interpretation of the results, approved the final version of the manuscript, and assume responsibility for the adherence of the trial to the protocol and for the accuracy and completeness of the data and analyses reported. No funder had any role in the design, conduct, analysis, or interpretation of the trial or in the writing of this report. Rosuvastatin was purchased (see the Supplementary Appendix).

\section{ELIGIBILITY}

Men and women who were 18 years of age or older and were scheduled to undergo elective coronary-artery bypass grafting (CABG), surgical aortic-valve replacement, or both were eligible if they were in sinus rhythm and were not taking antiarrhythmic medication (other than betablockers). Patients were excluded if they had moderate or severe mitral-valve disease or known renal dysfunction (creatinine level, $>2.3 \mathrm{mg}$ per deciliter [200 $\mu \mathrm{mol}$ per liter]) or had contraindications to statin therapy. All the participants provided written informed consent before enrollment.

\section{ENROLLMENT AND RANDOMIZATION}

Eligible patients underwent transthoracic echocardiography for the evaluation of left ventricular ejection fraction and left atrial size, and blood samples were obtained to measure concentrations of troponin I, N-terminal pro-brain natriuretic peptide (NT-proBNP), creatinine, lowdensity lipoprotein (LDL) cholesterol, and highsensitivity C-reactive protein. Any prescribed statin therapy was stopped, and patients were then randomly assigned to receive rosuvastatin at a dose of $20 \mathrm{mg}$ once daily or matching placebo tablets for up to 8 days before surgery and for 5 days thereafter (see the Methods section in the Supplementary Appendix).

\section{ASSESSMENT OF EFFECTS OF ROSUVASTATIN}

The two prespecified coprimary outcomes were postoperative atrial fibrillation and perioperative myocardial injury. Postoperative atrial fibrillation was detected by continuous Holter electrocardiographic monitoring within 5 days after surgery, and perioperative myocardial injury was 
assessed according to the area under the curve of troponin I release (a method of assessment that has been validated against magnetic resonance measurements of myocardial injury after cardiac surgery ${ }^{22}$ and that has been used in previous trials to evaluate perioperative myocardial injury $\left.{ }^{9,12}\right)$, as derived from blood samples obtained 6, 24, 48, and 120 hours after surgery.

Secondary outcomes were postoperative atrial fibrillation diagnosed clinically, alternative measures of myocardial injury, major in-hospital cardiovascular events and death, duration of ICU and hospital stay, the low cardiac output syndrome, pleural effusion, infection, left ventricular ejection fraction at discharge, acute kidney injury, and blood biomarkers. Postoperative myocardial infarction was diagnosed according to Thygesen et al., ${ }^{23}$ and acute kidney injury according to the Acute Kidney Injury Network criteria. ${ }^{24}$ Additional details are provided in the Methods section in the Supplementary Appendix.

\section{STATISTICAL ANALYSIS}

On the basis of data from previous trials, we estimated that the postoperative incidence of atrial fibrillation in the control (placebo) group would be approximately $35 \% .{ }^{25}$ We originally calculated that 1000 patients would need to undergo randomization in order for the trial to have more than $80 \%$ power, at a significance level of 0.05 , to detect a relative difference of $25 \%$ in the incidence of atrial fibrillation and a relative difference of $15 \%$ in the area under the curve of troponin I release. As recruitment progressed, however, the blinded rate of atrial fibrillation in the two trial groups combined was found to be approximately $20 \%$. Consequently, the sample was increased to approximately 1900 patients in order for the trial to retain at least $80 \%$ power, at a significance level of 0.05 , to detect a relative difference of $30 \%$ in the rate of atrial fibrillation.

We used odds ratios and 95\% confidence intervals for between-group comparisons of the primary outcome of atrial fibrillation and for other dichotomous outcomes and log-rank timeto-event methods for assessing the duration of ICU stay and total hospital stay. ${ }^{26}$ For the coprimary outcome of myocardial injury, analysis of covariance (ANCOVA) was used to compare the mean log area under the curve of troponin I release between 6 hours and 120 hours after surgery, with adjustment for the troponin I concen- tration at baseline. ANCOVA was also used to compare other biomarkers and echocardiographic measurements after surgery, with adjustment for baseline values. Analyses were performed on the log scale for the concentrations of creatinine, peak troponin I, NT-proBNP, and high-sensitivity C-reactive protein and then transformed back to the original scale as geometric means.

All the comparisons were performed according to the intention-to-treat principle. For dichotomous outcomes, patients with missing data were assumed not to have had the outcome. Missing values for biomarkers and echocardiographic measures (Fig. S1 in the Supplementary Appendix) were estimated by means of multiple imputation, with 10 replicate sets and combination across sets with the use of Rubin's methods. ${ }^{27}$ Consistency of these results with analyses that used alternative complete-case methods ${ }^{28}$ was assessed.

Prespecified analyses of the coprimary outcomes were performed in subgroups defined according to age at baseline ( $\leq 60$ years vs. $>60$ years), sex, previous statin use (yes vs. no), troponin I concentration $(\leq 0.04 \mathrm{ng}$ per milliliter vs. $>0.04 \mathrm{ng}$ per milliliter), duration of preoperative randomly assigned regimen ( $\leq 2$ days vs. $>2$ days), type of surgery (on-pump vs. off-pump; CABG vs. aortic-valve replacement), and postoperative use of nonsteroidal antiinflammatory drugs (NSAIDs) or glucocorticoids (yes vs. no). Observed effects in different subgroups were compared with the use of chi-square tests for heterogeneity. The approach that we used for an updated meta-analysis of the previously published randomized trials is described in the Methods section in the Supplementary Appendix.

\section{RESULTS}

\section{TRIAL PARTICIPANTS}

From September 2011 through October 2013, we screened 5429 patients who were scheduled to undergo elective cardiac surgery. A total of 2721 patients did not meet the inclusion criteria for the trial, 340 declined to take part, and 446 were not recruited for other reasons (Fig. S1 in the Supplementary Appendix). The characteristics at baseline of the 1922 patients who underwent randomization are shown in Table 1.

After randomization, surgery was cancelled for 48 patients (28 patients in the rosuvastatin group and 20 in the placebo group; $2 \%$ of the 
enrolled patients). A total of $87 \%$ of the patients underwent CABG surgery (including 3\% who, in addition, had aortic-valve replacement), 10\% underwent aortic-valve replacement alone, and $1 \%$ underwent some other type of surgery; $55 \%$ of the operations were on-pump (Table S2 in the Supplementary Appendix). The trial regimen was started up to and including 2 days before the day of surgery in 1131 of the 1922 patients (59\%) who underwent randomization and more than 2 days and up to 8 days before surgery in 743 (39\%). Among the 1874 randomly assigned patients who underwent surgery, adherence to the assigned regimen was $92 \%$ in each group.

The concentration of LDL cholesterol was reduced from baseline in both groups after surgery. However, the concentrations of both LDL cholesterol and high-sensitivity C-reactive protein were significantly lower in patients assigned to rosuvastatin than in those assigned to placebo $(\mathrm{P}<0.001)$ (Table 2). After surgery, $92 \%$ of the patients received beta-blockers and $60 \%$ received NSAIDs or dexamethasone (typically 5 to $10 \mathrm{mg}$ for 1 to 2 days) (Table S2 in the Supplementary Appendix).

\section{PRIMARY OUTCOMES}

Continuous Holter electrocardiographic recordings for 5 days after surgery were available for 1834 of the 1874 patients (98\%) who underwent surgery, with no difference between the trial groups in the percentage of patients who completed the 5 days of monitoring (Fig. S1 in the Supplementary Appendix). Postoperative atrial fibrillation was detected in 400 patients and was sustained in 368 (92\%). Rosuvastatin was not associated with a lower incidence of postoperative atrial fibrillation as compared with placebo; 203 patients $(21 \%)$ in the rosuvastatin group and $197(20 \%)$ in the placebo group had postoperative atrial fibrillation (odds ratio, 1.04; 95\% confidence interval [CI], 0.84 to $1.30 ; \mathrm{P}=0.72$ ) (Fig. 1).

Rosuvastatin did not have a significant effect on the incidence of postoperative atrial fibrillation in any of the prespecified subgroups (Fig. 1). In particular, rosuvastatin was not associated with a lower incidence of postoperative atrial fibrillation as compared with placebo among the 743 patients who started the trial regimen more than 2 days preoperatively (Fig. 1) or, in a nonprespecified analysis, among the 449 patients who started the regimen 4 to 8 days preopera-
Table 1. Characteristics of the Patients at Baseline."

\begin{tabular}{|c|c|c|}
\hline Characteristic & $\begin{array}{l}\text { Rosuvastatin } \\
(\mathrm{N}=960)\end{array}$ & $\begin{array}{r}\text { Placebo } \\
(\mathrm{N}=962)\end{array}$ \\
\hline \multicolumn{3}{|l|}{ Age } \\
\hline Mean - yr & $59.3 \pm 9.4$ & $59.5 \pm 9.5$ \\
\hline \multicolumn{3}{|l|}{ Distribution — no. (\%) } \\
\hline$\leq 60 \mathrm{yr}$ & $516(53.8)$ & $504(52.4)$ \\
\hline$>60 \mathrm{yr}$ & $444(46.2)$ & $458(47.6)$ \\
\hline Female sex - no. (\%) & $194(20.2)$ & $205(21.3)$ \\
\hline Body-mass index & $25.7 \pm 3.2$ & $25.7 \pm 3.1$ \\
\hline CKD-EPI estimated GFR - $\mathrm{ml} / \mathrm{min} / 1.73 \mathrm{~m}^{2} \mathrm{t}$ & $90 \pm 15$ & $90 \pm 15$ \\
\hline Current smoking — no. (\%) & $229(23.9)$ & $245(25.5)$ \\
\hline \multicolumn{3}{|l|}{ Medical history — no. (\%) } \\
\hline Hypertension & $621(64.7)$ & $614(63.8)$ \\
\hline Myocardial infarction & $282(29.4)$ & $274(28.5)$ \\
\hline Stroke or transient ischemic attack & $115(12.0)$ & $119(12.4)$ \\
\hline Peripheral arterial disease & $23(2.4)$ & $19(2.0)$ \\
\hline Heart failure & $46(4.8)$ & $37(3.8)$ \\
\hline Chronic obstructive pulmonary disease & $5(0.5)$ & $14(1.5)$ \\
\hline Diabetes mellitus & $310(32.3)$ & $291(30.2)$ \\
\hline Chronic kidney disease & $10(1.0)$ & $8(0.8)$ \\
\hline Contrast agent within previous $2 \mathrm{wk}$ & $383(39.9)$ & $379(39.4)$ \\
\hline \multicolumn{3}{|l|}{ Current or recent medication - no. (\%)』 } \\
\hline Beta-blocker & $813(84.7)$ & $804(83.6)$ \\
\hline NSAID or glucocorticoid & $16(1.7)$ & $7(0.7)$ \\
\hline Insulin & $140(14.6)$ & $158(16.4)$ \\
\hline Antiplatelet or anticoagulant & $792(82.5)$ & $779(81.0)$ \\
\hline Calcium-channel blocker & $445(46.4)$ & $424(44.1)$ \\
\hline ACE inhibitor or ARB & $385(40.1)$ & $384(39.9)$ \\
\hline Nitrate & $798(83.1)$ & $800(83.2)$ \\
\hline Diuretic agent & $197(20.5)$ & $213(22.1)$ \\
\hline Statin & $321(33.4)$ & $332(34.5)$ \\
\hline \multicolumn{3}{|l|}{ Scheduled surgery — no. (\%) } \\
\hline On-pump procedure & $508(52.9)$ & $515(53.5)$ \\
\hline Off-pump procedure & $422(44.0)$ & $429(44.6)$ \\
\hline CABG & $832(86.7)$ & $838(87.1)$ \\
\hline Aortic-valve replacement & $117(12.2)$ & $119(12.4)$ \\
\hline
\end{tabular}

* Plus-minus values are means \pm SD. The characteristics were well balanced between the trial groups at baseline; nominal $P$ values were greater than 0.05 for between-group differences in all the characteristics listed in the table, except for chronic obstructive pulmonary disease (nominal $P=0.04$; difference was not significant after allowance for multiple testing). ACE denotes angiotensinconverting enzyme, ARB angiotensin-receptor blocker, CABG coronary-artery bypass grafting, and NSAID nonsteroidal antiinflammatory drug.

$\uparrow$ The body-mass index is the weight in kilograms divided by the square of the height in meters.

$\$$ The glomerular filtration rate (GFR) was estimated with the Chronic Kidney Disease Epidemiology Collaboration (CKD-EPI) equation.

$\int$ Recent medication included antiplatelet agents, which are routinely stopped 5 to 7 days before surgery. 


\begin{tabular}{|c|c|c|c|}
\hline Variable & $\begin{array}{l}\text { Rosuvastatin } \\
(\mathrm{N}=960)\end{array}$ & $\begin{array}{l}\text { Placebo } \\
(\mathrm{N}=962)\end{array}$ & P Value \\
\hline \multicolumn{4}{|l|}{ LDL cholesterol - mg/dl } \\
\hline Baseline & $82.6 \pm 0.9$ & $80.2 \pm 0.9$ & - \\
\hline At $48 \mathrm{hr}{ }^{\dagger}$ & $37.1 \pm 0.5$ & $49.5 \pm 0.5$ & $<0.001$ \\
\hline At $120 \mathrm{hr} \boldsymbol{\gamma}^{-}$ & $49.9 \pm 0.6$ & $77.7 \pm 0.6$ & $<0.001$ \\
\hline \multicolumn{4}{|l|}{ Creatinine $-\mathrm{mg} / \mathrm{dl}$} \\
\hline Baseline & $0.85 \pm 0.01$ & $0.85 \pm 0.01$ & - \\
\hline At $48 \mathrm{hr} \uparrow$ & $1.02 \pm 0.01$ & $0.99 \pm 0.01$ & 0.007 \\
\hline At $120 \mathrm{hr}+$ & $0.87 \pm 0.01$ & $0.83 \pm 0.01$ & 0.001 \\
\hline \multicolumn{4}{|l|}{ Postsurgery troponin I concentration } \\
\hline Ratio of peak postsurgery value to baseline value & $237 \pm 12$ & $230 \pm 12$ & 0.66 \\
\hline Peak troponin I concentration within first $24 \mathrm{hr}-\mathrm{ng} / \mathrm{ml}$ & $2.6 \pm 0.1$ & $2.6 \pm 0.1$ & 0.89 \\
\hline \multicolumn{4}{|l|}{$\mathrm{N}$-terminal pro-brain natriuretic peptide $-\mathrm{pg} / \mathrm{ml}$} \\
\hline Baseline & $944 \pm 42$ & $951 \pm 42$ & - \\
\hline At $48 \mathrm{hr} \uparrow$ & $7545 \pm 161$ & $7416 \pm 164$ & 0.58 \\
\hline High-sensitivity C-reactive protein at $120 \mathrm{hr}$ - mg/liter & $50.9 \pm 0.9$ & $57.2 \pm 1.0$ & $<0.001$ \\
\hline \multicolumn{4}{|l|}{ Soluble CD40 ligand — pg/ml } \\
\hline Baseline & $648 \pm 23$ & $590 \pm 20$ & - \\
\hline At $48 \mathrm{hr}+$ & $260 \pm 11$ & $267 \pm 13$ & 0.65 \\
\hline \multicolumn{4}{|l|}{ Left atrial size $-\mathrm{cm}$} \\
\hline Baseline & $3.57 \pm 0.01$ & $3.58 \pm 0.01$ & - \\
\hline At discharge $\dagger$ & $3.38 \pm 0.01$ & $3.40 \pm 0.01$ & 0.21 \\
\hline \multicolumn{4}{|l|}{ Left ventricular ejection fraction — \% } \\
\hline Baseline & $60.5 \pm 0.3$ & $61.0 \pm 0.3$ & - \\
\hline At discharge $\dagger^{\dagger}$ & $59.3 \pm 0.2$ & $59.2 \pm 0.2$ & 0.65 \\
\hline \multicolumn{4}{|l|}{ Major in-hospital adverse event — no. (\%) } \\
\hline Atrial fibrillation $\mathbb{S}$ & $149(15.5)$ & $117(12.2)$ & 0.03 \\
\hline Arrhythmia other than atrial fibrillation & $427(44.5)$ & $431(44.8)$ & 0.89 \\
\hline Low cardiac output syndrome & $28(2.9)$ & $28(2.9)$ & 0.99 \\
\hline Pleural effusion & $84(8.8)$ & $95(9.9)$ & 0.40 \\
\hline Stroke & $5(0.5)$ & $5(0.5)$ & 1.00 \\
\hline Myocardial infarction & $37(3.9)$ & $41(4.3)$ & 0.65 \\
\hline Heart failure & $75(7.8)$ & $72(7.5)$ & 0.79 \\
\hline Infection & $80(8.3)$ & $90(9.4)$ & 0.43 \\
\hline Death & $3(0.3)$ & $1(0.1)$ & 0.34 \\
\hline \multicolumn{4}{|l|}{ Acute kidney injury at $48 \mathrm{hr} \mathbf{9}$} \\
\hline Stage 1 & $202(21.0)$ & $168(17.5)$ & 0.047 \\
\hline Stage 2 & $22(2.3)$ & $10(1.0)$ & 0.04 \\
\hline Stage 3 & $13(1.4)$ & $8(0.8)$ & 0.28 \\
\hline Any & 237 (24.7) & $186(19.3)$ & 0.005 \\
\hline
\end{tabular}




\begin{tabular}{|c|c|c|c|}
\hline Variable & $\begin{array}{l}\text { Rosuvastatin } \\
(\mathrm{N}=960)\end{array}$ & $\begin{array}{c}\text { Placebo } \\
(\mathrm{N}=962)\end{array}$ & P Value \\
\hline \multicolumn{4}{|l|}{ Time after surgery } \\
\hline To discharge from intensive care unit $-\mathrm{hr}$ & & & 0.17 \\
\hline Median & 42 & 41 & \\
\hline Interquartile range & $21-71$ & $21-69$ & \\
\hline To discharge from hospital — days & & & 0.18 \\
\hline Median & 7 & 7 & \\
\hline Interquartile range & $7-9$ & $7-9$ & \\
\hline
\end{tabular}

* Plus-minus values are arithmetic means \pm SE for low-density lipoprotein (LDL) cholesterol, left atrial size, and left ventricular ejection fraction; plus-minus values are geometric means with approximate \pm SE for the creatinine level, the ratio of the peak postsurgery value to the baseline value for the troponin I concentration, the peak troponin I concentration within the first 24 hours, and the levels of $\mathrm{N}$-terminal pro-brain natriuretic peptide, high-sensitivity C-reactive protein, and soluble CD40 ligand. Median and interquartile range values are product-limit estimates. To convert the values for cholesterol to millimoles per liter, multiply by 0.02586 . To convert the values for creatinine to micromoles per liter, multiply by 88.4 .

$\dagger$ The estimates and $\mathrm{P}$ values were derived from analysis of covariance with adjustment, where available, for the baseline value. Any missing data were imputed with the use of multiple imputation.

$\dagger$ The measurement was not prespecified.

$\int$ Atrial fibrillation was identified by means of routine electrocardiography or assessment of symptoms rather than by Holter monitoring.

ๆ Acute kidney injury at 48 hours was defined as an increase from baseline in the creatinine level of $0.3 \mathrm{mg}$ or more per deciliter $(30 \mu \mathrm{mol}$ per liter) or an increase by a factor of at least 1.5 to less than 2 (stage 1 ); an increase from baseline in the creatinine level by a factor of 2 to 3 (stage 2); or an increase from baseline in the creatinine level by a factor of more than 3 , a rise to a creatinine level of at least $4.0 \mathrm{mg}$ per deciliter ( $350 \mu \mathrm{mol}$ per liter), or the initiation of renal-replacement therapy (stage 3$) \cdot{ }^{24}$

tively. The incidence of atrial fibrillation was also not significantly lower with rosuvastatin than with placebo in other exploratory subgroup analyses, which included patients with a bodymass index (the weight in kilograms divided by the square of the height in meters) of more than 25 , those with a history of myocardial infarction, or those with a left ventricular ejection fraction of $55 \%$ or less.

For the coprimary outcome of perioperative myocardial injury, at least three of the four scheduled postsurgery measurements of the troponin I concentration were available for 1809 patients (97\%) who underwent surgery, with no between-group difference in the percentage of patients with at least three measurements (Fig. S1 in the Supplementary Appendix). Rosuvastatin had no significant effect on troponin I release after surgery; the area under the troponin I-release curve was $102 \mathrm{ng} \times$ hour per milliliter in the rosuvastatin group and $100 \mathrm{ng} \times$ hour per milliliter in the placebo group (between-group difference,
1\%; $95 \% \mathrm{CI},-9$ to $13 ; \mathrm{P}=0.80$ ) (Fig. 2). Analyses of the coprimary outcomes were not materially altered by adjustment for the characteristics at baseline.

Rosuvastatin also had no significant effect on secondary outcomes related to myocardial injury (Table 2). In addition, rosuvastatin was not associated with a significantly lower risk of myocardial injury than was placebo in any of the prespecified or retrospectively investigated subgroups. (For additional details on subgroups analyses, see Figs. S2, S3, and S4 in the Supplementary Appendix.)

\section{OTHER ADVERSE OUTCOMES}

In-hospital adverse outcomes were monitored until discharge. Atrial fibrillation was identified clinically by means of routine electrocardiography or assessment of symptoms in 16\% of the patients in the rosuvastatin group, as compared with $12 \%$ of those in the placebo group $(\mathrm{P}=0.03)$ (Table 2). Plasma creatinine levels were well 


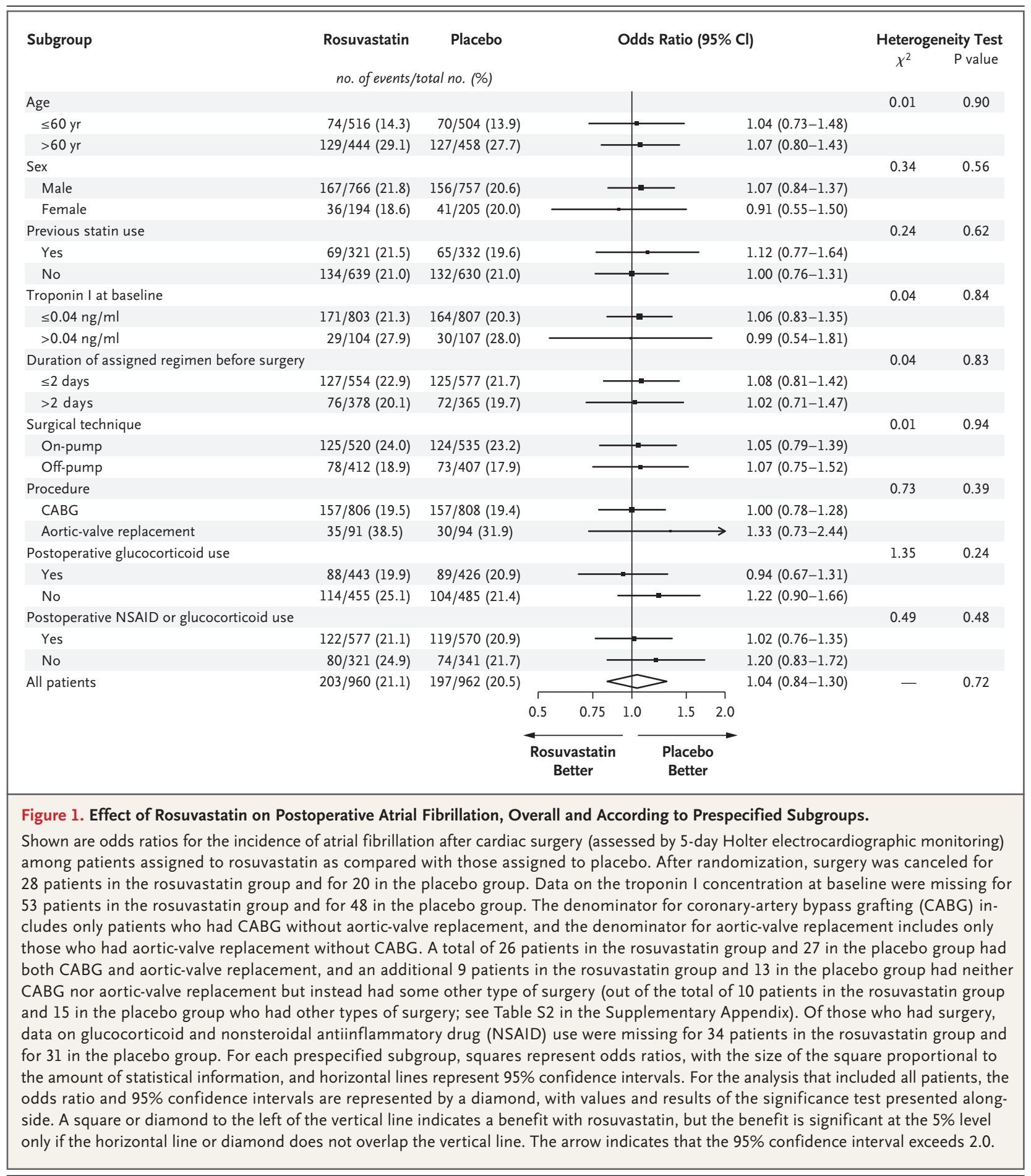

balanced at baseline but increased to a significantly greater extent among patients in the rosuvastatin group than among patients in the placebo group at 48 hours after surgery, and this excess was maintained up to 5 days (Table 2).
Acute kidney injury was significantly more common in the rosuvastatin group than in the placebo group (absolute $[ \pm S E]$ excess, $5.4 \pm 1.9$ percentage points; $\mathrm{P}=0.005)$; most of this excess was stage 1 acute kidney injury, but there was also 
a significant excess of stage 2 or 3 acute kidney injury (absolute excess, $1.8 \pm 0.7$ percentage points; $\mathrm{P}=0.02$ ) (Table 2).

Otherwise, there were no significant betweengroup differences in the rates of recorded postoperative serious adverse events, in the duration of ICU stay or hospital stay, or in echocardiographic variables (Table 2). The concentration of NT-proBNP at 48 hours was significantly increased from baseline in both groups, but the concentration of NT-proBNP and of the soluble CD40 ligand at 48 hours did not differ significantly between the trial groups.

\section{DISCUSSION}

In the STICS trial, the incidence of postoperative atrial fibrillation and the extent of perioperative myocardial injury, as assessed by the cumulative release of troponin I, did not differ between patients assigned to receive perioperative rosuvastatin and those assigned to receive placebo. On the basis of the confidence intervals for these randomized comparisons, our findings rule out rosuvastatin producing proportional reductions of more than 15 to $20 \%$ in the incidence of atrial fibrillation and of more than $10 \%$ in the release of troponin I. In addition, an excess of acute kidney injury was observed with perioperative rosuvastatin therapy.

An updated meta-analysis of the results from previously published randomized trials of perioperative statin therapy ${ }^{9-16,29-33}$ showed an approximate halving of the incidence of postoperative atrial fibrillation with statins as compared with placebo (Fig. S5 in the Supplementary Appendix). However, those trials involved small numbers of patients and had other important limitations (Table S1 in the Supplementary Appendix). For example, only four of the trials prespecified postoperative atrial fibrillation as an outcome, ${ }^{11,13,15,30}$ and only the Atorvastatin for Reduction of Myocardial Dysrhythmia after Cardiac Surgery (ARMYDA-3) trial ${ }^{13}$ included an intention-to-treat analysis of the effect on atrial fibrillation as detected in a systematic and blinded manner. Furthermore, a recent systematic Cochrane review of those trials showed evidence of selective reporting and publication bias. ${ }^{18}$ The suggestion that perioperative statin therapy might be associated with less severe myocardial damage derives from one randomized, placebo-controlled trial involv-

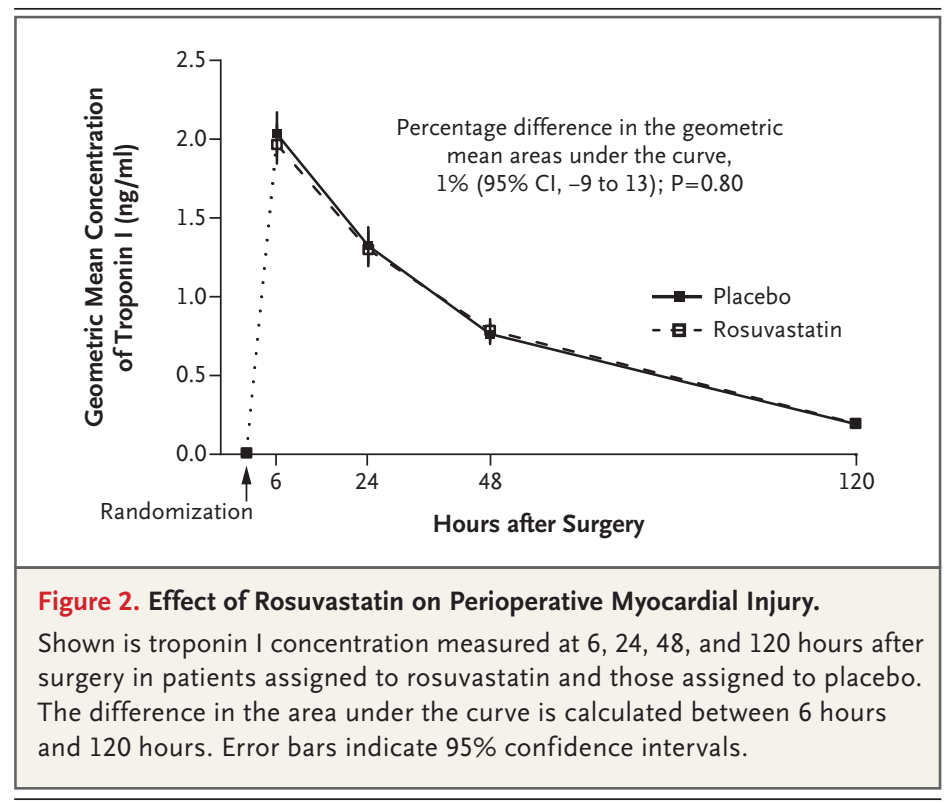

ing 200 patients undergoing CABG surgery ${ }^{9}$ and from a nonblinded randomized trial involving 151 patients undergoing noncoronary cardiac surgery, which also reported improved left ventricular function. ${ }^{12}$ Consequently, the large differences in outcomes reported in these trials may not provide a reliable indication of the real effects of perioperative statin therapy. ${ }^{34,35}$

By contrast, the STICS trial involved more patients and, of most relevance, more cases of atrial fibrillation than in all the previous trials combined. We recorded high levels of adherence to the assigned regimen, evaluated the prespecified outcomes systematically in a blinded manner, and compared outcomes between the trial groups on an intention-to-treat basis. The patients recruited in our trial were younger and had a lower prevalence of previous myocardial infarction and a higher average left ventricular ejection fraction, as well as a higher rate of betablocker use, than the patients in the ARMYDA-3 trial, ${ }^{13}$ all of which are likely to have contributed to the lower incidence of postoperative atrial fibrillation that we observed. Nevertheless, there was no evidence that perioperative rosuvastatin therapy produced beneficial effects among the large numbers of patients in high-risk subgroups that were included in our trial (Fig. S2 in the Supplementary Appendix).

A potential limitation of this trial is that the average duration of the randomly assigned regimen before surgery was shorter than the duration 
that was planned in most previous trials (although the actual preoperative duration of the regimen was reported in only one of those trials ${ }^{13}$ ). However, statins are known to exert rapid antiinflammatory and antioxidant effects in patients undergoing cardiac surgery. ${ }^{4,8}$ Moreover, even among the 449 patients who underwent randomization and started the regimen 4 to 8 days before surgery in our trial, there was no significant between-group difference in the incidence of atrial fibrillation or myocardial injury (Figs. S2 and S4 in the Supplementary Appendix).

The previous trials included only patients who had not been taking statin therapy before cardiac surgery; among the 1269 such patients in our trial, there was no beneficial effect of starting statin therapy on the incidence of postoperative atrial fibrillation or myocardial injury. In our trial, 653 patients were taking statin therapy at the time of randomization (a rate that is consistent with reported rates of statin use among patients with atherosclerotic disease in China $^{36}$ ); among these patients, the finding that there was no benefit from continuing statin therapy versus stopping it before surgery is directly relevant to current practice and indicates that continuing statin therapy during the perioperative period does not prevent in-hospital postoperative complications.

The lack of benefit of statin therapy in our trial might be related to the ethnic group of the trial population or to the specific drug (rosuvastatin) tested. However, previous trials have suggested that perioperative statin therapy was beneficial both in whites and in Southeast Asians, with the latter constituting approximately one third of the patients in the previously published trials, and, although atorvastatin was tested in most of the trials, benefits have also been reported with pravastatin, simvastatin, and rosuvastatin (Fig. S5 in the Supplementary Appendix).

Given the lack of good evidence of beneficial effects of perioperative statin therapy in our trial, the adverse effects on renal function warrant careful consideration. Rosuvastatin therapy resulted in significantly higher plasma creatinine levels than placebo at 48 hours after surgery, and this difference was maintained at 5 days. In parallel, there was a significant excess of acute kidney injury with rosuvastatin. Only two trials of perioperative statin therapy have included serial postoperative creatinine levels, ${ }^{12,14}$ and the significantly lower levels at 24 and 48 hours among the patients assigned to statin in their pooled results ${ }^{37}$ were driven primarily by the results of a nonblinded study involving Chinese patients undergoing noncoronary cardiac surgery. ${ }^{12}$ A meta-analysis of five trials that reported acute kidney injury after cardiac surgery in a total of 467 patients did not indicate any effect of statin therapy. ${ }^{37}$ Although the adverse effects on renal function that we observed may relate to the patients' ethnic group or to the use of rosuvastatin (which has been observed in other contexts to increase the incidence of proteinuria ${ }^{38}$ but not the creatinine level or the incidence of kidney injury ${ }^{39}$ ), a class effect of statin therapy in patients undergoing cardiac surgery cannot be ruled out.

In conclusion, despite promising observations in experimental studies and small clinical trials, we found that perioperative statin therapy did not prevent postoperative atrial fibrillation or perioperative myocardial damage in patients undergoing elective cardiac surgery. There were also no beneficial effects on postoperative left ventricular function or on the incidence of major cardiovascular adverse events, but acute kidney injury was more common with rosuvastatin.

\footnotetext{
Supported by the British Heart Foundation, the European Network for Translational Research in Atrial Fibrillation of the European Commission Seventh Framework Program, the Oxford Biomedical Research Centre, and the U.K. Medical Research Council and by a small unrestricted grant from AstraZeneca.

Disclosure forms provided by the authors are available with the full text of this article at NEJM.org.

We thank the patients who agreed to take part in the trial; the cardiac surgery teams in the Department of Cardiac Surgery, Fuwai Hospital, Beijing; the research support staff in the ChinaOxford Center for International Health Research, Fuwai Hospital; and the technicians in the Wolfson Laboratories of the Clinical Trial Service Unit and Epidemiological Studies Unit, Oxford, United Kingdom.
}

REFERENCES

1. LaPar DJ, Crosby IK, Rich JB, et al. A 2. Maesen B, Nijs J, Maessen J, Allessie contemporary cost analysis of postopera- $\quad \mathrm{M}$, Schotten U. Post-operative atrial fibriltive morbidity after coronary artery by- lation: a maze of mechanisms. Europace pass grafting with and without concomi- 2012;14:159-74.

tant aortic valve replacement to improve 3. Kim YM, Kattach H, Ratnatunga C, patient quality and cost-effective care. Pillai R, Channon KM, Casadei B. AssoAnn Thorac Surg 2013;96:1621-7.
Pillai R, Channon KM, Casadei B. Asso-
ciation of atrial nicotinamide adenine di- nucleotide phosphate oxidase activity with the development of atrial fibrillation after cardiac surgery. J Am Coll Cardiol 2008;51:68-74.

4. Antoniades C, Demosthenous $M$, Reilly S, et al. Myocardial redox state predicts in-hospital clinical outcome after 
cardiac surgery effects of short-term preoperative statin treatment. J Am Coll Cardiol 2012;59:60-70.

5. Halonen J, Halonen P, Järvinen O, et al. Corticosteroids for the prevention of atrial fibrillation after cardiac surgery: a randomized controlled trial. JAMA 2007;297: 1562-7.

6. Imazio M, Brucato A, Ferrazzi P, et al. Colchicine for prevention of postpericardiotomy syndrome and postoperative atrial fibrillation: the COPPS-2 randomized clinical trial. JAMA 2014;312:1016-23. 7. Pinho-Gomes AC, Reilly S, Brandes RP, Casadei B. Targeting inflammation and oxidative stress in atrial fibrillation: role of 3-hydroxy-3-methylglutaryl-coenzyme a reductase inhibition with statins. Antioxid Redox Signal 2014;20:1268-85.

8. Antoniades C, Bakogiannis C, Leeson P, et al. Rapid, direct effects of statin treatment on arterial redox state and nitric oxide bioavailability in human atherosclerosis via tetrahydrobiopterin-mediated endothelial nitric oxide synthase coupling. Circulation 2011;124:335-45.

9. Mannacio VA, Iorio D, De Amicis V, Di Lello F, Musumeci F. Effect of rosuvastatin pretreatment on myocardial damage after coronary surgery: a randomized trial. J Thorac Cardiovasc Surg 2008;136:1541-8. 10. Caorsi C, Pineda F, Munoz C. Pravastatin immunomodulates IL-6 and C-reactive protein, but not IL-1 and TNF-alpha, in cardio-pulmonary bypass. Eur Cytokine Netw 2008;19:99-103.

11. Sun Y, Ji Q, Mei Y, et al. Role of preoperative atorvastatin administration in protection against postoperative atrial fibrillation following conventional coronary artery bypass grafting. Int Heart 2011;52:7-11.

12. Almansob MA, Xu B, Zhou L, et al. Simvastatin reduces myocardial injury undergoing noncoronary artery cardiac surgery: a randomized controlled trial. Arterioscler Thromb Vasc Biol 2012;32: 2304-13.

13. Patti G, Chello M, Candura D, et al. Randomized trial of atorvastatin for reduction of postoperative atrial fibrillation in patients undergoing cardiac surgery: results of the ARMYDA-3 (Atorvastatin for Reduction of MYocardial Dysrhythmia After cardiac surgery) study. Circulation 2006;114:1455-61.

14. Tamayo E, Alvarez FJ, Alonso O, et al. Effects of simvastatin on systemic inflammatory responses after cardiopulmonary bypass. J Cardiovasc Surg (Torino) 2009; 50:687-94.

15. Song YB, On YK, Kim JH, et al. The effects of atorvastatin on the occurrence of postoperative atrial fibrillation after off-pump coronary artery bypass grafting surgery. Am Heart J 2008;156(2):373.e9-16. 16. Baran C, Durdu S, Dalva K, et al. Effects of preoperative short term use of atorvastatin on endothelial progenitor cells after coronary surgery: a randomized, controlled trial. Stem Cell Rev 2012; 8:963-71.

17. Kuhn EW, Liakopoulos OJ, Stange S, et al. Preoperative statin therapy in cardiac surgery: a meta-analysis of 90,000 patients. Eur J Cardiothorac Surg 2014;45:17-26. 18. Kuhn EW, Slottosch I, Wahlers T, Liakopoulos OJ. Preoperative statin therapy for patients undergoing cardiac surgery. Cochrane Database Syst Rev 2015;8: CD008493.

19. Hillis LD, Smith PK, Anderson JL, et al. 2011 ACCF/AHA Guideline for Coronary Artery Bypass Graft Surgery: a report of the American College of Cardiology Foundation/American Heart Association Task Force on Practice Guidelines: developed in collaboration with the American Association for Thoracic Surgery, Society of Cardiovascular Anesthesiologists, and Society of Thoracic Surgeons. J Am Coll Cardiol 2011;58(24):e123-210.

20. The Task Force for the Management of Atrial Fibrillation of the European Society of Cardiology. Guidelines for the management of atrial fibrillation. Europace 2010;12:1360-420.

21. January CT, Wann LS, Alpert JS, et al. 2014 AHA/ACC/HRS guideline for the management of patients with atrial fibrillation: a report of the American College of Cardiology/American Heart Association Task Force on Practice Guidelines and the Heart Rhythm Society. J Am Coll Cardiol 2014;64(21):e1-76.

22. Selvanayagam JB, Pigott D, Balacumaraswami L, Petersen SE, Neubauer S, Taggart DP. Relationship of irreversible myocardial injury to troponin I and creatine kinase-MB elevation after coronary artery bypass surgery: insights from cardiovascular magnetic resonance imaging. J Am Coll Cardiol 2005;45:629-31.

23. Thygesen K, Alpert JS, Jaffe AS, et al. Third universal definition of myocardial infarction. Eur Heart J 2012;33:2551-67. 24. Mehta RL, Kellum JA, Shah SV, et al. Acute Kidney Injury Network: report of an initiative to improve outcomes in acute kidney injury. Crit Care 2007;11:R31.

25. Chen WT, Krishnan GM, Sood N, Kluger J, Coleman CI. Effect of statins on atrial fibrillation after cardiac surgery: a duration- and dose-response meta-analysis. J Thorac Cardiovasc Surg 2010;140: 364-72.

26. Peto R, Pike MC, Armitage $\mathrm{P}$, et al. Design and analysis of randomized clini- cal trials requiring prolonged observation of each patient. II. Analysis and examples. Br J Cancer 1977;35:1-39.

27. Rubin DB. Multiple imputation for nonresponse in surveys. New York: John Wiley, 1987.

28. Little RJ, D'Agostino R, Cohen ML, et al. The prevention and treatment of missing data in clinical trials. $\mathrm{N}$ Engl J Med 2012;367:1355-60.

29. Chello M, Patti G, Candura D, et al. Effects of atorvastatin on systemic inflammatory response after coronary bypass surgery. Crit Care Med 2006;34:660-7.

30. Ji Q, Mei Y, Wang X, et al. Effect of preoperative atorvastatin therapy on atrial fibrillation following off-pump coronary artery bypass grafting. Circ J 2009;73: 2244-9.

31. Antoniades C, Bakogiannis C, Tousoulis $\mathrm{D}$, et al. Preoperative atorvastatin treatment in CABG patients rapidly improves vein graft redox state by inhibition of Rac1 and NADPH-oxidase activity. Circulation 2010;122:Suppl:S66-73.

32. Spadaccio C, Pollari F, Casacalenda A, et al. Atorvastatin increases the number of endothelial progenitor cells after cardiac surgery: a randomized control study. J Cardiovasc Pharmacol 2010;55:30-8.

33. Vukovic PM, Maravic-Stojkovic VR, Peric MS, et al. Steroids and statins: an old and a new anti-inflammatory strategy compared. Perfusion 2011;26:31-7.

34. Pereira TV, Horwitz RI, Ioannidis JP. Empirical evaluation of very large treatment effects of medical interventions. JAMA 2012;308:1676-84

35. Roberts I, Ker K, Edwards P, Beecher D, Manno D, Sydenham E. The knowledge system underpinning healthcare is not fit for purpose and must change. BMJ 2015; 350:h2463.

36. Li J, Chen YP, Li X, et al. Use of secondary preventive medications in patients with atherosclerotic disease in urban China: a cross-sectional study of 16,860 patients. Chin Med J (Engl) 2012;125:4361-7.

37. Lewicki M, Ng I, Schneider AG. HMG CoA reductase inhibitors (statins) for preventing acute kidney injury after surgical procedures requiring cardiac bypass. Cochrane Database Syst Rev 2015;3:CD010480. 38. Savarese G, Musella F, Volpe M, Paneni F, Perrone-Filardi P. Effects of atorvastatin and rosuvastatin on renal function: a meta-analysis. Int J Cardiol 2013;167: 2482-9.

39. Ridker PM, Danielson E, Fonseca FAH, et al. Rosuvastatin to prevent vascular events in men and women with elevated C-reactive protein. N Engl J Med 2008; 359:2195-207.

Copyright (c) 2016 Massachusetts Medical Society. 